\title{
A Supply Chain Network Perspective for Electric Power Generation, Supply, Transmission, and Consumption
}

\author{
Anna Nagurney and Dmytro Matsypura \\ Department of Finance and Operations Management \\ Isenberg School of Management \\ University of Massachusetts \\ Amherst, Massachusetts 01003
}

May, 2004; revised January 2005

Appears in Optimisation, Econometric and Financial Analysis

E. J. Kontoghiorghes and C. Gatu, Editors, (2006), Springer, Berlin, Germany, pp. 3-27.

\begin{abstract}
A supply chain network perspective for electric power production, supply, transmission, and consumption is developed. The model is sufficiently general to handle the behavior of the various decision-makers, who operate in a decentralized manner and include power generators, power suppliers, the transmitters, as well as the consumers associated with the demand markets. The optimality conditions are derived, along with the equilibrium state for the electric power supply chain network. The finite-dimensional variational inequality formulation of the equilibrium state is derived, whose solution yields the equilibrium electric power flows transacted between the tiers of the supply chain network as well as the nodal prices. The variational inequality formulation is utilized to provide qualitative properties of the equilibrium electric power flow and price patterns and to propose a computational scheme. The algorithm is then applied to compute the solutions to several numerical examples.
\end{abstract}

Key Words: electric power, supply chains, networks, variational inequalities, game theory 


\section{Introduction}

The electric power industry in the United States, as well as abroad, is undergoing a transformation from a regulated to a competitive industry. Whereas power generation was once dominated by vertically integrated investor-owned utilities who owned many of the generation capacity, transmission, and distribution facilities, the electric power industry today is characterized by many new companies that produce and market wholesale and retail electric power. In the United States, for example, several factors have made these changes both possible and necessary. First, technological advances have altered the economics of power production. For example, new gas-fired combined cycle power plants are more efficient and less costly than older coal-fired power plants. In addition, technological advances in electricity transmission equipment have made possible the economic transmission of power over long distances so that customers can now be more selective in choosing an electricity supplier. Secondly, between 1975 and 1985, residential electricity prices and industrial electricity prices in the US rose $13 \%$ and $28 \%$ in real terms, respectively (US Energy Information Administration (2000)).

Furthermore, the effects of the Public Utilities Regulatory Policies Act of 1978, which encouraged the development of nonutility power producers that used renewable energy to generate power, demonstrated that traditional vertically integrated electric utilities were not the only source of reliable power. Moreover, numerous legislative initiatives have been undertaken by the federal government in order to stimulate the development and strengthening of competitive wholesale power markets. As a consequence, by December 1, 2003, 1310 companies were eligible to sell wholesale power at market-based rates in the US (statistics available at http://www.eia.doe.gov).

The dramatic increase in the number of market participants trading over the past few years, as well as changes to electricity trading patterns have made system reliability more difficult to maintain. The North American Electric Reliability Council (NERC) reported that, "[in recent years] the adequacy of the bulk power transmission system has been challenged to support the movement of power in unprecedented amounts and in unexpected directions" (North American Electric Reliability Council (1998)). Moreover, a US Department of Energy Task Force noted that "there is a critical need to be sure that reliability is not taken for 
granted as the industry restructures, and thus does not fall through the cracks" (Secretary of Energy Advisory Board's (SEAB) Task Force on Electric System Reliability (1998)).

These concerns have helped to stimulate research activity in the area of electric power supply systems modeling and analysis during the past decade. Several models have been proposed that allow for more decentralization in the markets (see, e.g., Schweppe et al. (1988), Hogan (1992), Chao and Peck (1996), and Wu et al. (1996)). Some researchers have suggested different variations of the models depending on the electric power market organizational structure (see, for example, Hobbs (2001)). A wide range of models has been proposed for simulating the interaction of competing generation companies who price strategically (see Kahn (1998) and Hobbs, Metzler, and Pang (2000)), as well as those that simulate the exercising of market power on linearized dc networks based on a flexible representation of interactions of competing generating firms (Day, Hobbs, and Pang (2002)).

Nevertheless, despite all the research and analytical efforts, on August 14, 2003, large portions of the Midwest, the Northeastern United States, and Ontario, Canada, experienced an electric power blackout. The blackout left approximately 50 million people without electricity and affected 61,800 megawatts of electric load (U.S.-Canada Power System Outage Task Force (2003)). In addition, two significant outages during the month of September 2003 occurred abroad: one in England and one, initiated in Switzerland, that cascaded over much of Italy. The scale of these recent power outages has shown that the reliability of the existing power systems is not adequate and that the latest changes in electric power markets require deep and thorough analysis.

In this paper, we propose what we believe is a novel approach to the modeling and analysis of electric power markets. In particular, we develop a supply chain network model for electric power generation, supply, transmission, and consumption, which allows for decentralized decision-making, and which differs from recent models (see, e.g., Jing-Yuan and Smeers (1999), Takriti, Krasenbrink, and Wu (2000), Boucher and Smeers (2001), and Daxhelet and Smeers (2001)) in that, first and foremost, we consider several different types of decisionmakers and model their behavior and interactions explicitly. Moreover, we allow for not only the computation of electric power flows but also the prices associated with the various transactions between the tiers of decision-makers in the electric power supply chain network. 
Finally, the functional forms that can be handled in our framework are not limited to linear and/or separable functions. For additional background on supply chain network modeling, analysis, and computations, as well as financial engineering, see the annotated bibliography by Guenes and Pardalos (2003). For an overview of electric power systems, see the book by Casazza and Delea (2003). For an edited volume on the deregulation of electric utilities, see Zaccour (1998). For additional background on game theory as it relates to electric power systems, see the edited volume by Singh (1999).

The supply chain network approach permits one to represent the interactions between decision-makers in the market for electric power in terms of network connections, flows, and prices. In addition, we consider noncooperative behavior of decision-makers in the same tier of the supply chain network (such as, for example, the generators; the suppliers, and the demand markets) as well as cooperative behavior between tiers. Furthermore, this approach makes it possible to take advantage of the network topology (which is not limited to a specific number of generators, suppliers, transmitters, and/or demand markets) for computational purposes. Finally, it provides a framework from which a variety of extensions can be constructed to include, among other elements, multicriteria decision-making to incorporate environmental issues, risk and reliability elements, as well as stochastic components, and, in addition, the introduction of explicit dynamics and modeling of disequilibrium behavior.

The paper is organized as follows. In Section 2, we develop the model, describe the various decision-makers and their behavior, and construct the equilibrium conditions, along with the variational inequality formulation. The variables are the equilibrium prices, as well as the equilibrium electricity flows between the tiers of decision-makers. In Section 3, we derive qualitative properties of the equilibrium pattern, under appropriate assumptions, notably, the existence and uniqueness of a solution to the governing variational inequality. In Section 4, we propose an algorithm, which is then applied to several illustrative numerical examples in Section 5. We conclude the paper with Section 6 in which we summarize our results and suggest directions for future research. 


\section{The Supply Chain Network Model for Electric Power}

In this Section, we develop an electric power supply chain network model in which the decision-makers operate in a decentralized manner. In particular, we consider an electric power network economy in which goods and services are limited to electric energy and transmission services. We consider power generators, power suppliers (including power marketers, traders, and brokers), transmission service providers, and consumers (demand markets, or end users). A depiction of the supply chain network for electric power is given in Figure 1.

Power generators are those decision-makers who own and operate electric generating facilities or power plants. They produce electric power, which, is then sold to the power suppliers. The prices that generators charge for the electricity that they produce is determined by the competitive wholesale market. There is a total of $G$ power generators, depicted as the top tier nodes in Figure 1, with a typical power generator denoted by $g$. Power suppliers, in turn, bear a function of an intermediary. They buy electric power from power generators and sell to the consumers at different demand markets. We denote a typical supplier by $s$ and consider a total of $S$ power suppliers. Suppliers are represented by the second tier of nodes in the supply chain network in Figure 1.

Note that there is a link from each power generator to each supplier in the network in Figure 1 which represents that a supplier can buy energy from any generator on the wholesale market (equivalently, a generator can sell to any/all the suppliers). Note also that the links between the top tier and the second tier of nodes do not represent the physical connectivity of two particular nodes. Power suppliers do not physically possess electric power at any stage of the supplying process; they only hold the rights for the electric power. Hence, the link connecting a pair of such nodes in the supply chain is a decision-making connectivity link between that pair of nodes.

In order for electricity to be transmitted from a power generator to the point of consumption a transmission service is required. Hence, power suppliers need to buy the transmission services from the transmission service providers. Transmission service providers are those entities that own and operate the electric transmission and distribution systems. These are the companies that distribute electricity from generators via suppliers to demand markets (homes and businesses). Because transmission service providers do not make decisions as 


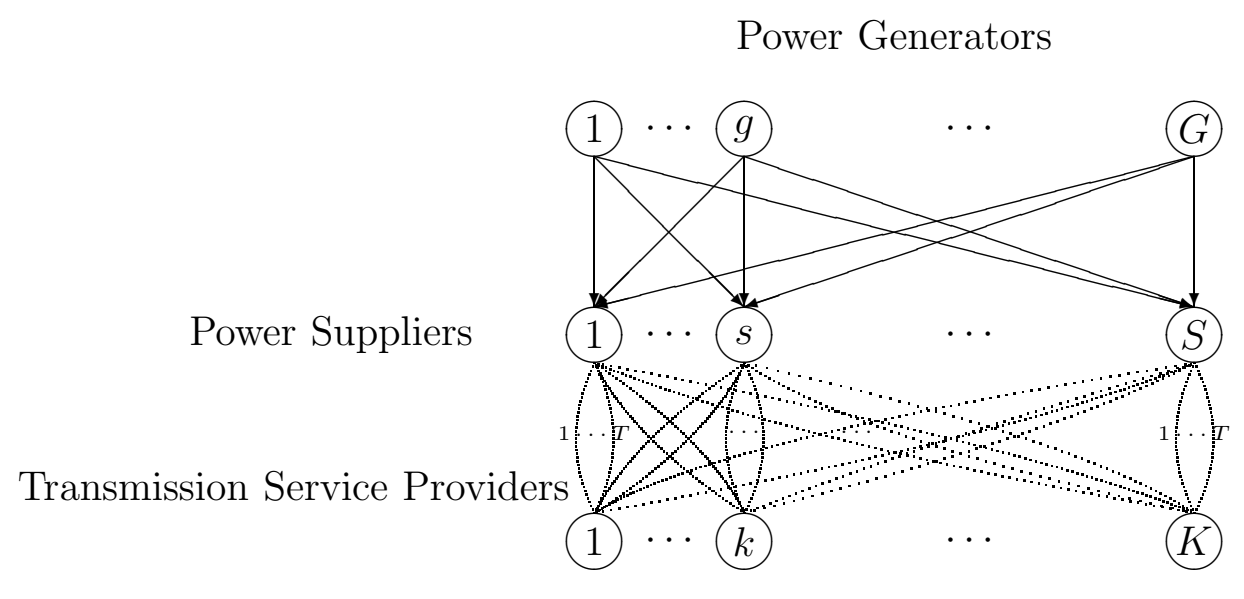

Demand Markets

Figure 1: The Electric Power Supply Chain Network

to where the electric power will be acquired and to whom it will be delivered, we do not include them in the model explicitly as nodes. Instead, their presence in the market is modeled as different modes of transaction (transmission modes) corresponding to distinct links connecting a given supplier node to a given demand market node in Figure 1. We assume that power suppliers cover the direct cost of the physical transaction of electric power from power generators to the demand markets and, therefore, have to make a decision as to from where to acquire the transmission services (and at what level).

We assume that there are $T$ transmission service providers operating in the supply chain network, with a typical transmission service provider denoted by $t$. For the sake of generality, we assume that every power supplier can transact with every demand market using any of the transmission service providers or any combination of them. Therefore, there are $T$ links joining every node in the middle tier of the network with every node at the bottom tier (see Figure 1). 
Finally, the last type of decision-maker in the model is the consumers or demand markets. They are depicted as the bottom tier nodes in Figure 1. These are the points of consumption of electric power. The consumers generate the demand that drives the generation and supply of the electric power in the entire system. There is a total of $K$ demand markets, with a typical demand market denoted by $k$, and distinguished from the others through the use of appropriate criteria, such as geographic location; the types of consumers; that is, whether they are businesses or households; etc. We assume a competitive electric power market, meaning that the demand markets can choose between different electric power suppliers (power marketers, brokers, etc.).

We also assume that a given power supplier negotiates with the transmission service providers and makes sure that the necessary electric power is delivered. These assumptions fit well into the main idea of the restructuring of the electric power industry that is now being performed in the US, the European Union, and many other countries (see http://www.ferc.gov and http://www.europarl.eu.int).

Clearly, in some situations, some of the links in the supply chain network for electric power in Figure 1 may not exist (due to, for example, various restrictions, regulations, etc.). This can be handled within our framework by eliminating the corresponding link for the supply chain network or (see further discussion below) assigning an appropriately high transaction cost associated with that link.

We now turn to the discussion of the behavior of each type of decision-maker and give the optimality conditions.

\section{The Behavior of Power Generators and their Optimality Conditions}

We first start with the description of the behavior of the power generators. Recall that power generators are those decision-makers in the network system, who own and operate electric generating facilities or power plants. They generate electric power and then sell it to the suppliers. Hence, one of the assumptions of our model is that power generators cannot trade directly with the demand markets.

Let $q_{g}$ denote the nonnegative amount of electricity in watts produced by electric power generator $g$ and let $q_{g s}$ denote the nonnegative amount of electricity (also in watts), being 
transacted from power generator $g$ to power supplier $s$. Note that $q_{g s}$ corresponds to the flow on the link joining node $g$ with node $s$ in Figure 1. We group the electric power production outputs for all power generators into the vector $q \in R_{+}^{G}$. Also, we group all the power flows associated with all the power generators to the suppliers into the column vector $Q^{1} \in R_{+}^{G S}$.

For power generator $g$, we assume, as given, a power generating cost function denoted by $f_{g}$, such that

$$
f_{g}=f_{g}(q), \quad \forall g
$$

All the power generating functions are assumed to be convex and continuously differentiable. Since generators compete for resources we allow for the general form (1). Of course, a special case is when $f_{g}=f_{g}\left(q_{g}\right)$.

Note that we allow each power generating cost function to depend not only on the amount of energy generated by a particular power generator, but also on the amount of energy generated by other power generators. This generalization allows one to model competition.

In addition, while the electric power is being transmitted from node $g$ to node $s$, there will be some transaction costs associated with the transmission process. Part of these costs will be covered by a power generator. Let $c_{g s}$ denote power generator $g$ 's transaction cost function associated with transmitting the electric power to supplier node $s$. We consider the general situation in which the transaction cost of a particular power generator is a function of not only the amount of power transacted by this particular power generator, but also of the amounts of power transacted by all other power generators (who may, for example, share the same physical line). Therefore, in order to represent the most general case, we have that

$$
c_{g s}=c_{g s}\left(Q^{1}\right), \quad \forall g, \forall s,
$$

and we assume that these functions are convex and continuously differentiable.

Each power generator $g$ faces the conservation of flow constraint given by:

$$
\sum_{s=1}^{S} q_{g s}=q_{g}
$$

that is, a power generator $g$ cannot ship out more electric power than he has produced. 
In view of (3) and (1), we may write, without any loss of generality that $f_{g}=f_{g}\left(Q^{1}\right)$, for all power generators $g ; g=1, \ldots, G$. Note that in our framework, as the production output reaches the capacity of a given generator then we expect the production cost to become very large (and, perhaps, even infinite).

\section{Optimization Problem of a Power Generator}

We assume that a typical power generator $g$ is a profit-maximizer. Let $\rho_{1 g s}^{*}$ denote the price that a power generator $g$ charges a power supplier $s$ per unit of electricity. We later in this section discuss how this price is arrived at. We allow the power generator to set different prices for different power suppliers. Hence, the optimization problem of the power generator $g$ can be expressed as follows:

$$
\text { Maximize } \quad \sum_{s=1}^{S} \rho_{1 g s}^{*} q_{g s}-f_{g}\left(Q^{1}\right)-\sum_{s=1}^{S} c_{g s}\left(Q^{1}\right)
$$

subject to:

$$
q_{g s} \geq 0, \quad \forall s .
$$

We assume that the power generators compete in noncooperative manner following the concepts of Nash $(1950,1951)$ (see also, e.g., Dafermos and Nagurney (1987)). Hence, each power generator seeks to determine his optimal strategy, that is, the generated outputs, given those of the other power generators. The optimality conditions of all power generators $g ; g=1, \ldots, G$, simultaneously, under the above assumptions (see also Bazaraa, Sherali, and Shetty (1993), Bertsekas and Tsitsiklis (1989), and Nagurney (1999)), can be compactly expressed as: determine $Q^{1 *} \in R_{+}^{G S}$ satisfying

$$
\sum_{g=1}^{G} \sum_{s=1}^{S}\left[\frac{\partial f_{g}\left(Q^{1 *}\right)}{\partial q_{g s}}+\frac{\partial c_{g s}\left(Q^{1 *}\right)}{\partial q_{g s}}-\rho_{1 g s}^{*}\right] \times\left[q_{g s}-q_{g s}^{*}\right] \geq 0, \quad \forall Q^{1} \in R_{+}^{G S} .
$$

Note that (6) is a variational inequality. Moreover, (6) has a very nice economic interpretation. Indeed, at optimality, if there is a positive flow of electric power between a generator/supplier pair, then the price charged is precisely equal to the sum of the marginal production cost plus the marginal transaction cost; if that sum exceeds the price, then there will be no electric power flow (and, thus, no transaction) between that pair. 


\section{The Behavior of Power Suppliers and their Optimality Conditions}

We now turn to the description of the behavior of the power suppliers. The term power supplier refers to power marketers, traders, and brokers, who arrange for the sale and purchase of the output of generators to other suppliers or load-serving entities, or in many cases, serve as load-serving entities themselves. They play a fundamental role in our model since they are responsible for acquiring electricity from power generators and delivering it to the demand markets. Therefore, power suppliers are involved in transactions with both power generators and the demand markets through transmission service providers.

A power supplier $s$ is faced with certain expenses, which may include, for example, the cost of licensing and the costs of maintenance. We refer collectively to such costs as an operating cost and denote it by $c_{s}$. Let $q_{s k}^{t}$ denote the amount of electricity being transacted between power supplier $s$ and demand market $k$ via the link corresponding to the transmission service provider $t$. We group all transactions associated with power supplier $s$ and demand market $k$ into the column vector $q_{s k} \in R_{+}^{T}$. We then further group all such vectors associated with all the power suppliers into a column vector $Q^{2} \in R_{+}^{S T K}$. For the sake of generality and to enhance the modeling of competition, we assume that

$$
c_{s}=c_{s}\left(Q^{1}, Q^{2}\right), \quad \forall s .
$$

We also assume that there is another type of cost that a power supplier may face, namely, transaction costs. As mentioned earlier, each power supplier is involved in transacting with both power generators and with the demand markets through transmission service providers. Therefore, there will be costs associated with each such transaction. These costs may include, for example, the expenses associated with maintaining the physical lines, if they belong to the power supplier, or the expenses associated with the transmission service which a power supplier has to purchase. In order to capture all possible scenarios, we will use a transaction cost function of a general form. Let $\hat{c}_{g s}$ denote the transaction cost associated with power supplier $s$ acquiring electric power from power generator $g$, where we asume that:

$$
\hat{c}_{g s}=\hat{c}_{g s}\left(Q^{1}\right), \quad \forall g, \forall s .
$$

Similarly, let $c_{s k}^{t}$ denote the transaction cost associated with power supplier $s$ transmitting 
electric power to demand market $k$ via transmission service provider $t$, where:

$$
c_{s k}^{t}=c_{s k}^{t}\left(Q^{2}\right), \quad \forall s, \forall k, \forall t
$$

We assume that all the above transaction cost functions are convex and continuously differentiable.

Note that in both (8) and (9), the cost functions are the functions of the total amount of electric power transmitted between the associated tiers of nodes and there is a perfectly logical reason for this. As was discussed before, the links in our model do not represent distinct physical lines, but, rather, they represent the possibility of transmitting electricity from one decision-maker to another. Therefore, we can very well have a case when different power generators share the same physical lines while transmitting the power to the same, or even different, power suppliers (demand markets). Although the actual transmission is being done from a generator to a demand market, the power supplier is the entity that negotiates for the transaction and covers the transmission costs. Hence, if a physical link is shared, the cost of transmission depends on the entire amount of energy that is going through. Our aim is to capture the most general situation and, hence, we allow the transmission cost functions to depend on the entire flow pattern between the associated tiers of nodes in the network.

Let $\rho_{2 s k}^{t}$ denote the price associated with the transaction from power supplier $s$ to demand market $k$ via transmission service provider $t$ and let $\rho_{2 s k}^{t *}$ denote the price actually charged (which we return to later in this section). The total amount of revenue the power supplier obtains from his transactions is equal to the sum over all the modes of transmission and all the demand markets of the price times the amount of electric power transacted with the demand market using the particular transmission mode. Indeed, the total revenue of power supplier $s$ can mathematically be expressed as follows:

$$
\sum_{k=1}^{K} \sum_{t=1}^{T} \rho_{2 s k}^{t *} q_{s k}^{t} .
$$

Before formulating an optimization problem of a typical power supplier, let us look closer at the transmission service providers and their role in the electric power supply chain network system. 


\section{Transmission Service Providers}

In order for electricity to be transmitted from a given power generator to the point of consumption a transmission service is required. Hence, power suppliers purchase the transmission services from the transmission service providers. Transmission service providers are those entities that own and operate the electric transmission and distribution systems. We assume that the price of transmission service depends on how far the electricity has to be transmitted; in other words, it can be different for different destinations (demand markets or consumers). We also let different transmission service providers have their services priced differently, which can be a result of a different level of quality of service, reliability of the service, etc.

In practice, an electric supply network is operated by an Independent System Operator (ISO) who operates as a disinterested, but efficient entity and does not own network or generation assets. His main objectives are: to provide independent, open and fair ac-

cess to transmission systems; to facilitate market-based, wholesale electricity rates; and to ensure the effective management and operation of the bulk power system in each region (http://www.isone.org). Therefore, the ISO does not control the electricity rates. Nevertheless, he makes sure that the prices of the transmission services are reasonable and not discriminatory. We model this aspect by having transmission service providers be pricetakers meaning that the price of their services is determined and cannot be changed by a transmission service provider himself. Hence, the price of transmission services is fixed. However, it is not constant, since it depends on the amount of electric power transmitted, the distance, etc., and may be calculated for each transmission line separately depending on the criteria listed above. Consequently, as was stated earlier, a transmission service provider does not serve as an explicit decision-maker in the complex network system.

\section{Optimization Problem of a Power Supplier}

Assuming that a typical power supplier $s$ is a profit-maximizer, we can express the optimization problem of power supplier $s$ as follows:

$$
\text { Maximize } \sum_{k=1}^{K} \sum_{t=1}^{T} \rho_{2 s k}^{t *} q_{s k}^{t}-c_{s}\left(Q^{1}, Q^{2}\right)-\sum_{g=1}^{G} \rho_{1 g s}^{*} q_{g s}-\sum_{g=1}^{G} \hat{c}_{g s}\left(Q^{1}\right)-\sum_{k=1}^{K} \sum_{t=1}^{T} c_{s k}^{t}\left(Q^{2}\right)
$$


subject to:

$$
\begin{gathered}
\sum_{k=1}^{K} \sum_{t=1}^{T} q_{s k}^{t} \leq \sum_{g=1}^{G} q_{g s} \\
q_{g s} \geq 0, \quad \forall g \\
q_{s k}^{t} \geq 0, \quad \forall k, \forall t .
\end{gathered}
$$

The objective function (11) represents the profit of power supplier $s$ with the first term denoting the revenue and the subsequent terms the various costs and payouts to the generators. Inequality (12) is a conservation of flow inequality which states that a power supplier $s$ cannot provide more electricity than he obtains from the power generators.

We assume that the power suppliers also compete in a noncooperative manner (as we assumed for the power generators). Hence, each power supplier seeks to determine his optimal strategy, that is, the input (accepted) and output flows, given those of the other power suppliers. The optimality conditions of all power suppliers $s ; s=1, \ldots, S$, simultaneously, under the above assumptions (see also Dafermos and Nagurney (1987) and Nagurney, Dong, and Zhang (2002)), can be compactly expressed as: determine $\left(Q^{1 *}, Q^{2 *}, \gamma^{*}\right) \in R_{+}^{S(G+K T+1)}$ satisfying

$$
\begin{gathered}
\sum_{s=1}^{S} \sum_{k=1}^{K} \sum_{t=1}^{T}\left[\frac{\partial c_{s}\left(Q^{1 *}, Q^{2 *}\right)}{\partial q_{s k}^{t}}+\frac{\partial c_{s k}^{t}\left(Q^{2 *}\right)}{\partial q_{s k}^{t}}-\rho_{2 s k}^{t *}+\gamma_{s}^{*}\right] \times\left[q_{s k}^{t}-q_{s k}^{t *}\right] \\
+\sum_{s=1}^{S} \sum_{g=1}^{G}\left[\frac{\partial c_{s}\left(Q^{1 *}, Q^{2 *}\right)}{\partial q_{g s}}+\frac{\partial \hat{c}_{g s}\left(Q^{1 *}\right)}{\partial q_{g s}}+\rho_{1 g s}^{*}-\gamma_{s}^{*}\right] \times\left[q_{g s}-q_{g s}^{*}\right] \\
+\sum_{s=1}^{S}\left[\sum_{g=1}^{G} q_{g s}^{*}-\sum_{k=1}^{K} \sum_{t=1}^{T} q_{s k}^{t *}\right] \times\left[\gamma_{s}-\gamma_{s}^{*}\right] \geq 0, \quad \forall\left(Q^{1}, Q^{2}, \gamma\right) \in R_{+}^{S(G+K T+1)},
\end{gathered}
$$

where $\gamma_{s}^{*}$ is the optimal Lagrange multiplier associated with constraint (12), and $\gamma$ is the corresponding $S$-dimensional vector of Lagrange multipliers.

Note that $\gamma_{s}^{*}$ serves as a "market-clearing" price in that, if positive, the electric power flow transacted out of supplier $s$ must be equal to that amount accepted by the supplier from all the power generators. Also, note that from (15) we can infer that if there is a positive flow $q_{g s}^{*}$, then $\gamma_{s}^{*}$ is precisely equal to the marginal operating cost of supplier $s$ plus 
the marginal cost associated with this transaction plus the price per unit of electric power paid by supplier $s$ to generator $g$.

\section{Equilibrium Conditions for the Demand Markets}

We now turn to the description of the equilibrium conditions for the demand markets. Let $\rho_{3 k}$ denote the price per unit of electric power associated with the demand market $k$. Note here that we allow the final price of electric power to be different at different demand markets. We assume that the demand for electric power at each demand market $k$ is elastic and depends not only on the price at the corresponding demand market but may, in general, also depend on the entire vector of the final prices in the supply chain network economy, that is,

$$
d_{k}=d_{k}\left(\rho_{3}\right)
$$

where $\rho_{3}=\left(\rho_{31}, \ldots, \rho_{3 k}, \ldots, \rho_{3 K}\right)^{T}$. This level of generality also allows one to facilitate the modeling of competition on the consumption side.

Let $\hat{c}_{s k}^{t}$ denote the unit transaction cost associated with obtaining the electric power at demand market $k$ from supplier $s$ via transmission mode $t$, where we assume that this transaction cost is continuous and of the general form:

$$
\hat{c}_{s k}^{t}=\hat{c}_{s k}^{t}\left(Q^{2}\right), \quad \forall s, \forall k, \forall t
$$

The equilibrium conditions associated with the transactions between power suppliers and

demand markets take the following form: We say that a vector $\left(Q^{2 *}, \rho_{3}^{*}\right) \in R_{+}^{K(S T+1)}$ is an equilibrium vector if for each $s, k, t$ :

$$
\rho_{2 s k}^{t *}+\hat{c}_{s k}^{t}\left(Q^{2 *}\right) \begin{cases}=\rho_{3 k}^{*}, & \text { if } \quad q_{s k}^{t *}>0 \\ \geq \rho_{3 k}^{*}, & \text { if } \quad q_{s k}^{t *}=0\end{cases}
$$

and

$$
d_{k}\left(\rho_{3}^{*}\right)\left\{\begin{array}{lll}
=\sum_{s=1}^{S} \sum_{t=1}^{T} q_{s k}^{t *}, & \text { if } & \rho_{3 k}^{*}>0 \\
\leq \sum_{s=1}^{S} \sum_{t=1}^{T} q_{s k}^{t *}, & \text { if } & \rho_{3 k}^{*}=0
\end{array}\right.
$$

Conditions (18) state that consumers at demand market $k$ will purchase the electric power from power supplier $s$, if the price charged by the power supplier plus the transaction cost 
does not exceed the price that the consumers are willing to pay for the electric power. Note that, according to (18), if the transaction costs are identically equal to zero, then the price faced by the consumers for the electric power is the price charged by the power supplier.

Condition (19), on the other hand, states that, if the price the consumers are willing to pay for the electric power at a demand market is positive, then the amount of the electric power transacted by the power suppliers with the consumers at the demand market is precisely equal to the demand. Conditions (18) and (19) are in concert with the ones in Nagurney, Dong, and Zhang (2002), and reflect, spatial price equilibrium (see also, e.g., Nagurney (1999)).

Note that the satisfaction of (18) and (19) is equivalent to the solution of the variational inequality given by: determine $\left(Q^{2 *}, \rho_{3}^{*}\right) \in R_{+}^{K(S T+1)}$, such that

$$
\begin{gathered}
\sum_{s=1}^{S} \sum_{k=1}^{K} \sum_{t=1}^{T}\left[\rho_{2 s k}^{t *}+\hat{c}_{s k}^{t}\left(Q^{2 *}\right)-\rho_{3 k}^{*}\right] \times\left[q_{s k}^{t}-q_{s k}^{t *}\right]+\sum_{k=1}^{K}\left[\sum_{s=1}^{S} \sum_{t=1}^{T} q_{s k}^{t *}-d_{k}\left(\rho_{3}^{*}\right)\right] \times\left[\rho_{3 k}-\rho_{3 k}^{*}\right] \geq 0 \\
\forall\left(Q^{2}, \rho_{3}\right) \in R_{+}^{K(S T+1)}
\end{gathered}
$$

\section{The Equilibrium Conditions for the Power Supply Chain Network}

In equilibrium, the amounts of electricity transacted between the power generators and the power suppliers must coincide with those that the power suppliers actually accept. In addition, the amounts of the electricity that are obtained by the consumers must be equal to the amounts that the power suppliers actually provide. Hence, although there may be competition between decision-makers at the same tier of nodes of the power supply chain network there must be, in a sense, cooperation between decision-makers associated with pairs of nodes (through positive flows on the links joining them). Thus, in equilibrium, the prices and product flows must satisfy the sum of the optimality conditions (6) and (15), and the equilibrium conditions (20). We make these relationships rigorous through the subsequent definition and variational inequality derivation.

\section{Definition 1: Electric Power Supply Chain Network Equilibrium}

The equilibrium state of the electric power supply chain network is one where the electric 
power flows between the tiers of the network coincide and the electric power flows and prices satisfy the sum of conditions (6), (15), and (20).

We now state and prove:

\section{Theorem 1: Variational Inequality Formulation of the Electric Power Supply Chain Network Equilibrium}

The equilibrium conditions governing the power supply chain network according to Definition 1 are equivalent to the solution of the variational inequality given by: determine $\left(Q^{1 *}, Q^{2 *}, \gamma^{*}, \rho_{3}^{*}\right) \in \mathcal{K}$ satisfying:

$$
\begin{gathered}
\sum_{g=1}^{G} \sum_{s=1}^{S}\left[\frac{\partial f_{g}\left(Q^{1 *}\right)}{\partial q_{g s}}+\frac{\partial c_{g s}\left(Q^{1 *}\right)}{\partial q_{g s}}+\frac{\partial c_{s}\left(Q^{1 *}, Q^{2 *}\right)}{\partial q_{g s}}+\frac{\partial \hat{c}_{g s}\left(Q^{1 *}\right)}{\partial q_{g s}}-\gamma_{s}^{*}\right] \times\left[q_{g s}-q_{g s}^{*}\right] \\
+\sum_{s=1}^{S} \sum_{k=1}^{K} \sum_{t=1}^{T}\left[\frac{\partial c_{s}\left(Q^{1 *}, Q^{2 *}\right)}{\partial q_{s k}^{t}}+\frac{\partial c_{s k}^{t}\left(Q^{2 *}\right)}{\partial q_{s k}^{t}}+\hat{c}_{s k}^{t}\left(Q^{2 *}\right)+\gamma_{s}^{*}-\rho_{3 k}^{*}\right] \times\left[q_{s k}^{t}-q_{s k}^{t *}\right] \\
+\sum_{s=1}^{S}\left[\sum_{g=1}^{G} q_{g s}^{*}-\sum_{k=1}^{K} \sum_{t=1}^{T} q_{s k}^{t *}\right] \times\left[\gamma_{s}-\gamma_{s}^{*}\right]+\sum_{k=1}^{K}\left[\sum_{s=1}^{S} \sum_{t=1}^{T} q_{s k}^{t *}-d_{k}\left(\rho_{3}^{*}\right)\right] \times\left[\rho_{3 k}-\rho_{3 k}^{*}\right] \geq 0, \\
\forall\left(Q^{1}, Q^{2}, \gamma, \rho_{3}\right) \in \mathcal{K},
\end{gathered}
$$

where $\mathcal{K} \equiv\left\{\left(Q^{1}, Q^{2}, \gamma, \rho_{3}\right) \mid\left(Q^{1}, Q^{2}, \gamma, \rho_{3}\right) \in R_{+}^{G S+T S K+S+K}\right\}$.

Proof: We first establish that the equilibrium conditions imply variational inequality (21). Indeed, summation of inequalities (6), (15), and (20), after algebraic simplifications, yields variational inequality (21).

We now establish the converse, that is, that a solution to variational inequality (21) satisfies the sum of conditions (6), (15), and (20), and is, hence, an equilibrium.

Consider inequality (21). Add term $\rho_{1 g s}^{*}-\rho_{1 g s}^{*}$ to the term in the first set of brackets (preceding the first multiplication sign). Similarly, add term $\rho_{2 s k}^{t *}-\rho_{2 s k}^{t *}$ to the term in the second set of brackets (preceding the second multiplication sign). The addition of such terms does not change (21) since the value of these terms is zero and yields:

$$
\sum_{g=1}^{G} \sum_{s=1}^{S}\left[\frac{\partial f_{g}\left(Q^{1 *}\right)}{\partial q_{g s}}+\frac{\partial c_{g s}\left(Q^{1 *}\right)}{\partial q_{g s}}+\frac{\partial c_{s}\left(Q^{1 *}, Q^{2 *}\right)}{\partial q_{g s}}+\frac{\partial \hat{c}_{g s}\left(Q^{1 *}\right)}{\partial q_{g s}}-\gamma_{s}^{*}+\rho_{1 g s}^{*}-\rho_{1 g s}^{*}\right] \times\left[q_{g s}-q_{g s}^{*}\right]
$$




$$
\begin{gathered}
+\sum_{s=1}^{S} \sum_{k=1}^{K} \sum_{t=1}^{T}\left[\frac{\partial c_{s}\left(Q^{1 *}, Q^{2 *}\right)}{\partial q_{s k}^{t}}+\frac{\partial c_{s k}^{t}\left(Q^{2 *}\right)}{\partial q_{s k}^{t}}+\hat{c}_{s k}^{t}\left(Q^{2 *}\right)+\gamma_{s}^{*}-\rho_{3 k}^{*}+\rho_{2 s k}^{t *}-\rho_{2 s k}^{t *}\right] \times\left[q_{s k}^{t}-q_{s k}^{t *}\right] \\
+\sum_{s=1}^{S}\left[\sum_{g=1}^{G} q_{g s}^{*}-\sum_{k=1}^{K} \sum_{t=1}^{T} q_{s k}^{t *}\right] \times\left[\gamma_{s}-\gamma_{s}^{*}\right]+\sum_{k=1}^{K}\left[\sum_{s=1}^{S} \sum_{t=1}^{T} q_{s k}^{t *}-d_{k}\left(\rho_{3}^{*}\right)\right] \times\left[\rho_{3 k}-\rho_{3 k}^{*}\right] \geq 0 \\
\forall\left(Q^{1}, Q^{2}, \gamma, \rho_{3}\right) \in \mathcal{K}
\end{gathered}
$$

which can be rewritten as:

$$
\begin{gathered}
\sum_{g=1}^{G} \sum_{s=1}^{S}\left[\frac{\partial f_{g}\left(Q^{1 *}\right)}{\partial q_{g s}}+\frac{\partial c_{g s}\left(Q^{1 *}\right)}{\partial q_{g s}}-\rho_{1 g s}^{*}\right] \times\left[q_{g s}-q_{g s}^{*}\right] \\
+\sum_{s=1}^{S} \sum_{k=1}^{K} \sum_{t=1}^{T}\left[\frac{\partial c_{s}\left(Q^{1 *}, Q^{2 *}\right)}{\partial q_{s k}^{t}}+\frac{\partial c_{s k}^{t}\left(Q^{2 *}\right)}{\partial q_{s k}^{t}}-\rho_{2 s k}^{t *}+\gamma_{s}^{*}\right] \times\left[q_{s k}^{t}-q_{s k}^{t *}\right] \\
+\sum_{s=1}^{S} \sum_{g=1}^{G}\left[\frac{\partial c_{s}\left(Q^{1 *}, Q^{2 *}\right)}{\partial q_{g s}}+\frac{\partial \hat{c}_{g s}\left(Q^{1 *}\right)}{\partial q_{g s}}+\rho_{1 g s}^{*}-\gamma_{s}^{*}\right] \times\left[q_{g s}-q_{g s}^{*}\right] \\
+\sum_{s=1}^{S}\left[\sum_{g=1}^{G} q_{g s}^{*}-\sum_{k=1}^{K} \sum_{t=1}^{T} q_{s k}^{t *}\right] \times\left[\gamma_{s}-\gamma_{s}^{*}\right] \\
+\sum_{s=1}^{S} \sum_{k=1}^{K} \sum_{t=1}^{T}\left[\rho_{2 s k}^{t *}+\hat{c}_{s k}^{t}\left(Q^{2 *}\right)-\rho_{3 k}^{*}\right] \times\left[q_{s k}^{t}-q_{s k}^{t *}\right]+\sum_{k=1}^{K}\left[\sum_{s=1}^{S} \sum_{t=1}^{T} q_{s k}^{t *}-d_{k}\left(\rho_{3}^{*}\right)\right] \times\left[\rho_{3 k}-\rho_{3 k}^{*}\right] \geq 0 \\
\forall\left(Q^{1}, Q^{2}, \gamma, \rho_{3}\right) \in \mathcal{K} .
\end{gathered}
$$

Inequality (23) is a sum of equilibrium conditions (6), (15), and (20). Therefore, the electric power flow and price pattern is an equilibrium according to Definition 1.

The variational inequality problem $(21)$ can be rewritten in standard variational inequality form (cf. Nagurney (1999)) as follows: determine $X^{*} \in \mathcal{K}$ satisfying

$$
\left\langle F\left(X^{*}\right)^{T}, X-X^{*}\right\rangle \geq 0, \quad \forall X \in \mathcal{K}
$$

where $X \equiv\left(Q^{1}, Q^{2}, \gamma, \rho_{3}\right)$, and $F(X) \equiv\left(F_{g s}, F_{s k}^{t}, F_{s}, F_{k}\right)_{g=1, \ldots, G ; s=1, \ldots, S ; t=1, \ldots, T ; k=1, \ldots, K}$, with the specific components of $F$ given by the functional terms preceding the multiplication signs in $(21)$, respectively. $\langle\cdot, \cdot\rangle$ denotes the inner product in $N$-dimensional Euclidian space where here $N=G S+S K T+S+K$. 
We now describe how to recover the prices associated with the first two tiers of nodes in the power supply chain network. Clearly, the components of the vector $\rho_{3}^{*}$ are obtained directly from the solution to variational inequality (21). In order to recover the second tier prices $\rho_{2}^{*}$ associated with the power suppliers one can (after solving variational inequality (21) for the particular numerical problem) either (cf. (18)) set $\rho_{2 s k}^{t *}=\rho_{3 k}^{*}-\hat{c}_{s k}^{t}\left(Q^{2 *}\right)$ for any $s, t, k$ such that $q_{s k}^{t *}>0$, or (cf. (15)) set $\rho_{2 s k}^{t *}=\frac{\partial c_{s}\left(Q^{1 *}, Q^{2 *}\right)}{\partial q_{s k}^{t}}+\frac{\partial c_{s k}^{t}\left(Q^{2 *}\right)}{\partial q_{s k}^{t}}+\gamma_{s}^{*}$ for any $s, t, k$ such that $q_{s k}^{t *}>0$.

Similarly, from (6) we can infer that the top tier prices comprising the vector $\rho_{1}^{*}$ can be recovered (once the variational inequality (21) is solved with particular data) in the following way: for any $g, s$ such that $q_{g s}^{*}>0$, set $\rho_{1 g s}^{*}=\frac{\partial f_{g}\left(Q^{1 *}\right)}{\partial q_{g s}}+\frac{\partial c_{g s}\left(Q^{1 *}\right)}{\partial q_{g s}}$ or, equivalently, from (15): set $\rho_{1 g s}^{*}=\gamma_{s}^{*}-\frac{\partial c_{s}\left(Q^{1 *}, Q^{2 *}\right)}{\partial q_{g s}}-\frac{\partial \hat{c}_{g s}\left(Q^{1 *}\right)}{\partial q_{g s}}$.

\section{Theorem 2}

The solution to the variational inequality (22) satisfies variational inequalities (6), (15), and (20) (separately) under the condition that vectors $\rho_{1}^{*}$ and $\rho_{2}^{*}$ are derived using the procedure described above.

Proof: Suppose that $\left(Q^{1 *}, Q^{2 *}, \gamma^{*}, \rho_{3}^{*}\right) \in \mathcal{K}$ is a solution to variational inequality (21). Variational inequality $(21)$ has to hold for all $\left(Q^{1}, Q^{2}, \gamma, \rho_{3}\right) \in \mathcal{K}$. Using the procedure for deriving vectors $\rho_{1}^{*}$ and $\rho_{2}^{*}$ one can get (23) from (21). Now, consider expression (23) from the proof of Theorem 1. If one lets $\gamma_{s}=\gamma_{s}^{*}, \rho_{3 k}=\rho_{3 k}^{*}$, and $q_{s k}^{t}=q_{s k}^{t *}$ for all $s, k$, and $t$ in (23), one obtains the following expression:

$$
\sum_{g=1}^{G} \sum_{s=1}^{S}\left[\frac{\partial f_{g}\left(Q^{1 *}\right)}{\partial q_{g s}}+\frac{\partial c_{g s}\left(Q^{1 *}\right)}{\partial q_{g s}}-\rho_{1 g s}^{*}\right] \times\left[q_{g s}-q_{g s}^{*}\right] \geq 0, \quad \forall Q^{1} \in R_{+}^{G S},
$$

which is exactly variational inequality (6) and, therefore, a solution to (21) also satisfies (6).

Similarly, if one lets $\rho_{3 k}=\rho_{3 k}^{*}$ for all $k, q_{s k}^{t}=q_{s k}^{t *}$ for all $s, k$, and $t$ in the fourth functional term (preceding the fourth multiplication sign), and also lets $q_{g s}=q_{g s}^{*}$ in the first functional term (preceding the first multiplication sign) in (24), one obtains the following expression:

$$
\sum_{s=1}^{S} \sum_{k=1}^{K} \sum_{t=1}^{T}\left[\frac{\partial c_{s}\left(Q^{1 *}, Q^{2 *}\right)}{\partial q_{s k}^{t}}+\frac{\partial c_{s k}^{t}\left(Q^{2 *}\right)}{\partial q_{s k}^{t}}-\rho_{2 s k}^{t *}+\gamma_{s}^{*}\right] \times\left[q_{s k}^{t}-q_{s k}^{t *}\right]
$$




$$
\begin{gathered}
+\sum_{s=1}^{S} \sum_{g=1}^{G}\left[\frac{\partial c_{s}\left(Q^{1 *}, Q^{2 *}\right)}{\partial q_{g s}}+\frac{\partial \hat{c}_{g s}\left(Q^{1 *}\right)}{\partial q_{g s}}+\rho_{1 g s}^{*}-\gamma_{s}^{*}\right] \times\left[q_{g s}-q_{g s}^{*}\right] \\
+\sum_{s=1}^{S}\left[\sum_{g=1}^{G} q_{g s}^{*}-\sum_{k=1}^{K} \sum_{t=1}^{T} q_{s k}^{t *}\right] \times\left[\gamma_{s}-\gamma_{s}^{*}\right] \geq 0, \quad \forall\left(Q^{1}, Q^{2}, \gamma\right) \in R_{+}^{S(G+K T+1)},
\end{gathered}
$$

which is exactly variational inequality (15) and, therefore, a solution to (21) also satisfies $(15)$.

Finally, if one lets $\gamma_{s}=\gamma_{s}^{*}, q_{g s}=q_{g s}^{*}$ for all $g$ and $s$, and also $q_{s k}^{t}=q_{s k}^{t *}$ for all $s, k$, and $t$ and substitutes these into the second functional term (preceding the second multiplication sign) in (23), one obtains the following expression:

$$
\begin{gathered}
\sum_{s=1}^{S} \sum_{k=1}^{K} \sum_{t=1}^{T}\left[\rho_{2 s k}^{t *}+\hat{c}_{s k}^{t}\left(Q^{2 *}\right)-\rho_{3 k}^{*}\right] \times\left[q_{s k}^{t}-q_{s k}^{t *}\right]+\sum_{k=1}^{K}\left[\sum_{s=1}^{S} \sum_{t=1}^{T} q_{s k}^{t *}-d_{k}\left(\rho_{3}^{*}\right)\right] \times\left[\rho_{3 k}-\rho_{3 k}^{*}\right] \geq 0 \\
\forall\left(Q^{2}, \rho_{3}\right) \in R_{+}^{K(S T+1)}
\end{gathered}
$$

which is exactly variational inequality (20) and, hence, a solution to (21) also satisfies (20).

We have, thus, established that a solution to variational inequality (21) also satisfies (6), (15), and (20) separately under the pricing mechanism described above. 


\section{Qualitative Properties}

In this Section, we provide some qualitative properties of the solution to variational inequality (24). In particular, we derive existence and uniqueness results.

Since the feasible set is not compact we cannot derive existence simply from the assumption of continuity of the functions. We can, however, impose a rather weak condition to guarantee existence of a solution pattern. Let

$$
\mathcal{K}_{b}=\left\{\left(Q^{1}, Q^{2}, \gamma, \rho_{3}\right) \mid 0 \leq Q^{1} \leq b_{1} ; 0 \leq Q^{2} \leq b_{2} ; 0 \leq \gamma \leq b_{3} ; 0 \leq \rho_{3} \leq b_{4}\right\},
$$

where $b=\left(b_{1}, b_{2}, b_{3}, b_{4}\right) \geq 0$ and $Q^{1} \leq b_{1}, Q^{2} \leq b_{2}, \gamma \leq b_{3}$, and $\rho_{3} \leq b_{4}$ means $q_{g s} \leq b_{1}$,

$q_{s k}^{t} \leq b_{2}, \gamma_{s} \leq b_{3}$, and $\rho_{3 k} \leq b_{4}$ for all $g, s, k$, and $t$. Then $\mathcal{K}_{b}$ is a bounded, closed, convex subset of $R_{+}^{G S+S K T+S+K}$. Therefore, the following variational inequality:

$$
\left\langle F\left(X^{b}\right)^{T}, X-X^{b}\right\rangle \geq 0, \quad \forall X \in \mathcal{K}_{b},
$$

admits at least one solution $X_{b} \in \mathcal{K}_{b}$, from the standard theory of variational inequalities, since $\mathcal{K}_{b}$ is compact and $F$ is continuous. Following Kinderlehrer and Stampacchia (1980) (see also Nagurney (1999)), we then have the following theorems:

\section{Theorem 3: Existence}

Variational inequality (24) (equivalently (21)) admits a solution if and only if there exists a vector $b>0$, such that variational inequality (26) admits a solution in $\mathcal{K}_{b}$ with

$$
Q^{1 b}<b_{1}, \quad Q^{2 b}<b_{2}, \quad \gamma^{b}<b_{3}, \quad \rho_{3}^{b}<b_{4} .
$$

\section{Theorem 4: Uniqueness}

Assume that conditions of Theorem 3 hold, that is, variational inequality (26) and, hence, variational inequality (24) admits at least one solution. Suppose that function $F(X)$ that enters variational inequality (24) is strictly monotone on $\mathcal{K}$, that is,

$$
\left\langle\left(F\left(X^{\prime}\right)-F\left(X^{\prime \prime}\right)\right)^{T}, X^{\prime}-X^{\prime \prime}\right\rangle>0, \quad \forall X^{\prime}, X^{\prime \prime} \in \mathcal{K}, \quad X^{\prime} \neq X^{\prime \prime} .
$$

Then the solution to variational inequality (24) is unique. 


\section{The Algorithm}

In this Section, an algorithm is presented that can be applied to solve any variational inequality problem in standard form (see (24)), that is: determine $X^{*} \in \mathcal{K}$, satisfying:

$$
\left\langle F\left(X^{*}\right)^{T}, X-X^{*}\right\rangle \geq 0, \quad \forall X \in \mathcal{K}
$$

The algorithm is guaranteed to converge provided that the function $F(X)$ that enters the variational inequality is monotone and Lipschitz continuous (and that a solution exists). The algorithm is the modified projection method of Korpelevich (1977) and it has been applied to solve a plethora of network equilibrium problems (see Nagurney and Dong (2002)).

We first provide a definition of a Lipschitz continuous function:

\section{Definition 2: Lipschitz Continuity}

A function $F(X)$ is Lipschitz continuous, if there exists a constant $L>0$ such that:

$$
\left\|F\left(X^{\prime}\right)-F\left(X^{\prime \prime}\right)\right\| \leq L\left\|X^{\prime}-X^{\prime \prime}\right\|, \quad \forall X^{\prime}, X^{\prime \prime} \in \mathcal{K}, \text { with } L>0
$$

The statement of the modified projection method is as follows, where $\mathcal{T}$ denotes an iteration counter:

\section{Modified Projection Method}

\section{Step 0: Initialization}

Set $X^{0} \in \mathcal{K}$. Let $\mathcal{T}=1$ and let $a$ be a scalar such that $0<a \leq \frac{1}{L}$, where $L$ is the Lipschitz continuity constant (cf. (29)).

\section{Step 1: Computation}

Compute $\bar{X}^{\mathcal{T}}$ by solving the variational inequality subproblem:

$$
\left\langle\bar{X}^{\mathcal{T}}+a F\left(X^{\mathcal{T}-1}\right)^{T}-X^{\mathcal{T}-1}, X-\bar{X}^{\mathcal{T}}\right\rangle \geq 0, \quad \forall X \in \mathcal{K}
$$




\section{Step 2: Adaptation}

Compute $X^{\mathcal{T}}$ by solving the variational inequality subproblem:

$$
\left\langle X^{\mathcal{T}}+a F\left(\bar{X}^{\mathcal{T}}\right)^{T}-X^{\mathcal{T}-1}, X-X^{\mathcal{T}}\right\rangle \geq 0, \quad \forall X \in \mathcal{K}
$$

\section{Step 3: Convergence Verification}

If $\max \left|X_{l}^{\mathcal{T}}-X_{l}^{\mathcal{T}-1}\right| \leq \epsilon$, for all $l$, with $\epsilon>0$, a prespecified tolerance, then stop; else, set $\mathcal{T}=: \mathcal{T}+1$, and go to Step 1 .

The following theorem states the convergence result for the modified projection method and is due to Korpelevich (1977).

\section{Theorem 5: Convergence}

Assume that the function that enters the variational inequality (21) (or (24)) has at least one solution and is monotone, that is,

$$
\left\langle\left(F\left(X^{\prime}\right)-F\left(X^{\prime \prime}\right)\right)^{T}, X^{\prime}-X^{\prime \prime}\right\rangle \geq 0, \quad \forall X^{\prime}, X^{\prime \prime} \in \mathcal{K}
$$

and Lipschitz continuous. Then the modified projection method described above converges to the solution of the variational inequality (21) or (24).

The realization of the modified projection method in the context of the electric power supply chain network model takes on a very elegant form for computational purposes. In particular, the feasible set $\mathcal{K}$ is a Cartesian product, consisting of only nonnegativity constraints on the variables which allows for the network structure to be exploited. Hence, the induced quadratic programming problems in (30) and (31) can be solved explicitly and in closed form using explicit formulae for the power flows between the tiers of the supply chain network, the demand market prices, and the optimal Lagrange multipliers.

Conditions for $F$ to be monotone and Lipschitz continuous can be obtained from the results in Nagurney, Dong, and Zhang (2002). 


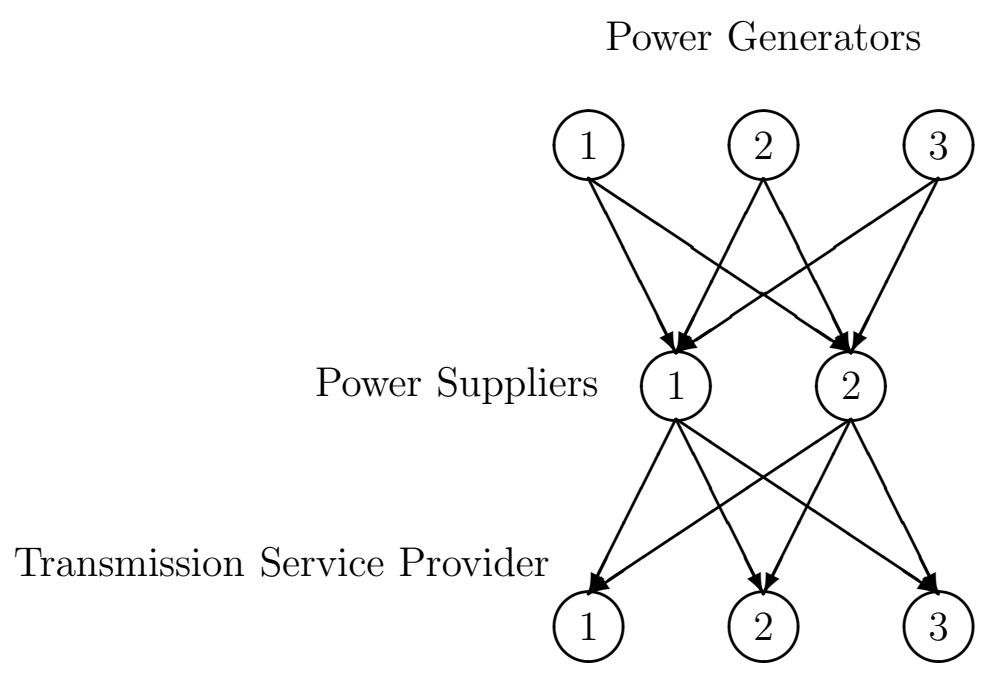

Demand Markets

Figure 2: Electric Power Supply Chain Network for the Numerical Examples

\section{Numerical Examples}

In this Section, we apply the modified projection method to several numerical examples. The modified projection method was implemented in FORTRAN and the computer system used was a Sun system located at the University of Massachusetts at Amherst.

The convergence criterion utilized was that the absolute value of the flows $\left(Q^{1}, Q^{2}\right)$ and the prices $\left(\gamma, \rho_{3}\right)$ between two successive iterations differed by no more than $10^{-4}$. For the examples, $a$ was set to .05 in the algorithm, except where noted otherwise. The numerical examples had the network structure depicted in Figure 2 and consisted of three power generators, two power suppliers, and three demand markets, with a single transmission service provider available to each power supplier.

The modified projection method was initialized by setting all variables equal to zero. 


\section{Example 1}

The power generating cost functions for the power generators were given by:

$$
f_{1}(q)=2.5 q_{1}^{2}+q_{1} q_{2}+2 q_{1}, \quad f_{2}(q)=2.5 q_{2}^{2}+q_{1} q_{2}+2 q_{2}, \quad f_{3}(q)=.5 q_{3}^{2}+.5 q_{1} q_{3}+2 q_{3} .
$$

The transaction cost functions faced by the power generators and associated with transacting with the power suppliers were given by:

$$
\begin{gathered}
c_{11}\left(Q^{1}\right)=.5 q_{11}^{2}+3.5 q_{11}, c_{12}\left(Q^{1}\right)=.5 q_{12}^{2}+3.5 q_{12}, c_{21}\left(Q^{1}\right)=.5 q_{21}^{2}+3.5 q_{21}, c_{22}\left(Q^{1}\right)=.5 q_{22}^{2}+3.5 q_{22}, \\
c_{31}\left(Q^{1}\right)=.5 q_{31}^{2}+2 q_{31}, \quad c_{32}\left(Q^{1}\right)=.5 q_{32}^{2}+2 q_{32} .
\end{gathered}
$$

The operating costs of the power generators, in turn, were given by:

$$
c_{1}\left(Q^{1}, Q^{2}\right)=.5\left(\sum_{i=1}^{2} q_{i 1}\right)^{2}, \quad c_{2}\left(Q^{1}, Q^{2}\right)=.5\left(\sum_{i=1}^{2} q_{i 2}\right)^{2} .
$$

The demand functions at the demand markets were:

$$
\begin{gathered}
d_{1}\left(\rho_{3}\right)=-2 \rho_{31}-1.5 \rho_{32}+1100, \quad d_{2}\left(\rho_{3}\right)=-2 \rho_{32}-1.5 \rho_{31}+1100, \\
d_{3}\left(\rho_{3}\right)=-2 \rho_{33}-1.5 \rho_{31}+1200,
\end{gathered}
$$

and the transaction costs between the power suppliers and the consumers at the demand markets were given by:

$$
\begin{array}{lll}
\hat{c}_{11}^{1}\left(Q^{2}\right)=q_{11}^{1}+5, & \hat{c}_{12}^{1}\left(Q^{2}\right)=q_{12}^{1}+5, & \hat{c}_{13}^{1}\left(Q^{2}\right)=q_{13}^{1}+5, \\
\hat{c}_{21}^{1}\left(Q^{2}\right)=q_{21}^{1}+5, & \hat{c}_{22}^{1}\left(Q^{2}\right)=q_{22}^{1}+5, & \hat{c}_{23}^{1}\left(Q^{2}\right)=q_{23}^{1}+5 .
\end{array}
$$

All other transaction costs were assumed to be equal to zero.

The modified projection method converged in 232 iterations and yielded the following equilibrium pattern:

$$
q_{11}^{*}=q_{12}^{*}=q_{21}^{*}=q_{22}^{*}=14.2762 ; \quad q_{31}^{*}=q_{32}^{*}=57.6051,
$$




$$
q_{11}^{1 *}=q_{12}^{1 *}=q_{21}^{1 *}=q_{22}^{1 *}=20.3861 ; \quad q_{31}^{1 *}=q_{32}^{1 *}=45.3861 .
$$

The vector $\gamma^{*}$ had components:

$$
\gamma_{1}^{*}=\gamma_{2}^{*}=277.2487
$$

and the demand prices at the demand markets were:

$$
\rho_{31}^{*}=\rho_{32}^{*}=302.6367 ; \quad \rho_{33}^{*}=327.6367 .
$$

It is easy to verify that the optimality/equilibrium conditions were satisfied with good accuracy.

\section{Example 2}

We then constructed the following variant of Example 1. We kept the data identical to that in Example 1 except that we that we changed the first demand function so that:

$$
d_{1}\left(\rho_{3}\right)=-2 \rho_{33}-1.5 \rho_{31}+1500
$$

The modified projection method converged in 398 iterations, yielding the following new equilibrium pattern:

$$
\begin{gathered}
q_{11}^{*}=q_{12}^{*}=q_{21}^{*}=q_{22}^{*}=19.5994 ; \quad q_{31}^{*}=q_{32}^{*}=78.8967, \\
q_{11}^{1 *}=q_{21}^{1 *}=118.0985
\end{gathered}
$$

and all other $q_{s k}^{1 *} \mathrm{~s}=0.0000$. The vector $\gamma^{*}$ had components:

$$
\gamma_{1}^{*}=\gamma_{2}^{*}=378.3891
$$

and the demand prices at the demand markets were:

$$
\rho_{31}^{*}=501.4873, \quad \rho_{32}^{*}=173.8850, \quad \rho_{33}^{*}=223.8850 .
$$

It is easy to verify that the optimality/equilibrium conditions were satisfied with good accuracy. 
Note that with the increased demand at demand market 1 as evidenced through the new demand function, the demand price at that market increased. This was the only demand market that had positive electric power flowing into it; the other two demand markets had zero electric power consumed.

\section{Example 3}

We then modified Example 2 as follows: The data were identical to that in Example 2 except that we changed the coefficient preceding the first term in the power generating function associated with the first power generator so that rather than having the term $2.5 q_{1}^{2}$ in $f_{1}(q)$ there was now the term $5 q_{1}^{2}$. We also changed $a$ to .03 since the modified projection method did not converge with $a=.05$. Note that $a$ must lie in a certain range, which is data-dependent, for convergence.

The modified projection method converged in 633 iterations, yielding the following new equilibrium pattern:

$$
\begin{gathered}
q_{11}^{*}=q_{12}^{*}=10.3716, \quad q_{21}^{*}=q_{22}^{*}=21.8956, \quad q_{31}^{*}=q_{32}^{*}=84.2407 . \\
q_{11}^{1 *}=q_{21}^{1 *}=116.5115,
\end{gathered}
$$

with all other $q_{s k}^{1 *} \mathrm{~s}=0.0000$.

The vector $\gamma^{*}$ had components:

$$
\gamma_{1}^{*}=\gamma_{2}^{*}=383.6027,
$$

and the demand prices at the demand markets were:

$$
\rho_{31}^{*}=505.1135, \quad \rho_{32}^{*}=171.1657, \quad \rho_{33}^{*}=221.1657 .
$$

As expected, since the power generating cost function associated with the first power generator increased, the power that he generated decreased; the power generated by the two other power generators, on the other hand, increased. Again, as in Example 2, there was no demand (at the computed equilibrium prices) at the second and third demand markets. 


\section{Example 4}

The fourth, and final example, was constructed as follows from Example 3. The data were all as in Example 3, but we now assumed that the demand functions were separable; hence, from each of the three demand market functions for electric power in Example 3, we eliminated the term not corresponding to the price at the specific market. In other words, the demand at demand market 1 only depended upon the price at demand market 1 ; the demand at demand market 2 only depended upon the demand at demand market 2; and the same held for the third demand market.

The modified projection method now converged in 325 iterations and yielded the following equilibrium electric power flow and price pattern:

$$
\begin{aligned}
& q_{11}^{*}=q_{12}^{*}=14.1801, \quad q_{21}^{*}=q_{22}^{*}=29.9358, \quad q_{31}^{*}=q_{32}^{*}=114.9917 \\
& q_{11}^{1 *}=q_{21}^{1 *}=111.3682, \quad q_{12}^{1 *}=q_{22}^{1 *}=11.3683, \quad q_{13}^{1 *}=q_{23}^{1 *}=36.3682 .
\end{aligned}
$$

The vector $\gamma^{*}$ had components:

$$
\gamma_{1}^{*}=\gamma_{2}^{*}=522.2619,
$$

whereas the equilibrium demand prices at the demand markets were now:

$$
\rho_{31}^{*}=638.6319, \quad \rho_{32}^{*}=538.6319, \quad \rho_{33}^{*}=563.6319 .
$$

Observe that since now there were no cross-terms in the demand functions, the electric power flows transacted between the suppliers and the demand markets were all positive. Of course, the incurred demands at both the second and third demand markets also increased. In addition, all the equilibrium flows from the power generators to the suppliers increased since there was increased demands at all the demand markets for electric power.

These numerical examples, although stylized, demonstrate the types of simulations that can be carried out. Indeed, one can easily investigate the effects on the equilibrium power flows and prices of such changes as: changes to the demand functions, to the power generating cost functions, as well as to the other cost functions. In addition, one can easily add or 
remove various decision-makers by changing the supply chain network structure (with the corresponding addition/removal of appropriate nodes and links) to investigate the effects of such market structure changes.

\section{Conclusions and Future Research}

In this paper, we proposed a theoretically rigorous framework for the modeling, qualitative analysis, and computation of solutions to electric power market flows and prices in an equilibrium context based on a supply chain network approach. The theoretical analysis was based on finite-dimensional variational inequality theory.

We modeled the behavior of the decision-makers, derived the optimality conditions as well as the governing equilibrium conditions which reflect competition among decision-makers (in a game-theoretic framework) at the same tier of nodes but cooperation between tiers of nodes. The framework allows for the handling of as many power generators, power suppliers, transmission service providers, and demand markets, as mandated by the specific application. Moreover, the underlying functions associated with electric power generation, transmission, as well as consumption can be nonlinear and non-separable. The formulation of the equilibrium conditions was shown to be equivalent to a finite-dimensional variational inequality problem. The variational inequality problem was then utilized to obtain qualitative properties of the equilibrium flow and price pattern as well as to propose a computational procedure for the numerical determination of the equilibrium electric power prices and flows.

In addition, we illustrated both the model and computational procedure through several numerical examples in which the electric power flows as well as the prices at equilibrium were computed.

As mentioned in the Introduction, there are many ways in which this basic foundational framework can be extended, notably, through the incorporation of multicriteria decisionmaking associated with the decision-makers (with, for example, such criteria as environmental impacts, reliability, risk, etc.), the introduction of stochastic components, as well as the introduction of dynamics to study the disequilibrium electric power flows and prices. 


\section{Acknowledgments}

The authors are grateful to the two anonymous referees and to the editor, Erricos Kontoghiorghes, for helpful comments and suggestions. This research was supported, in part, by an AT\&T Industrial Ecology Fellowship. This support is gratefully appreciated.

\section{References}

Bazaraa, M. S., Sherali, H. D. and C. M. Shetty (1993), Nonlinear Programming: Theory and Algorithms, John Wiley \& Sons, New York.

Bertsekas, D. P. and J. N. Tsitsiklis (1989), Parallel and Distributed Computation Numerical Methods, Prentice Hall, Englewood Cliffs, New Jersey.

Boucher, J. and Y. Smeers (2001), "Alternative Models of Restructured Electricity Systems Part 1: No Market Power," Operations Research 49, 821-838.

Casazza, J. and F. Delea (2003), Understanding Electric Power Systems, John Wiley \& Sons, New York.

Chao, H.-P. and S. Peck (1996), "A Market Mechanism for Electric Power Transmission," Journal of Regulatory Economics 10, 25-60.

Dafermos, S. and A. Nagurney (1987), "Oligopolistic and Competitive Behavior of Spatially Separated Markets," Regional Science and Urban Economics 17, 245-254.

Daxhelet, O. and Y. Smeers (2001), "Variational Inequality Models of Restructured Electric Systems," in Applications and Algorithms of Complementarity, M. C. Ferris, O. L. Mangasarian, and J. S. Pang, Editors, Kluwer Academic Publishers, Boston, Massachusetts.

Day, C. J., Hobbs, B. F. and Pang, J. S. (2002), "Oligopolistic Competition in Power Networks: A Conjectured Supply Function Approach," IEEE Transactions on Power Systems 17, 597-607.

Gabay, D. and H. Moulin (1980), "On the Uniqueness and Stability of Nash Equilibria in Noncooperative Games," in Applied Stochastic Control in Econometrics and Man- 
agement Science, A. Bensoussan, P. Kleindorfer, and C. S. Tapiero, Editors, NorthHolland, Amsterdam, The Netherlands, 271-294.

Guenes, J. and P. M. Pardalos, 2003. "Network Optimization in Supply Chain Management and Financial Engineering: An Annotated Bibliography," Networks 42, 66-84.

Hobbs, B. F. (2001), "Linear Complementarity Models of NashCournot Competition in Bilateral and POOLCO Power Markets," IEEE Transactions on Power Systems 16, 194202.

Hobbs, B. F., Metzler, C. and J. S. Pang (2000), "Strategic Gaming Analysis for Electric Power Networks: An MPEC Approach," IEEE Transactions on Power Systems 15, 638-645.

Hogan, W. (1992), "Contract Networks for Electric Power Transmission," Journal of Regulatory Economics 4, 211-242.

Hogan, W., Cadwalader, M. D., Harvey, S. M. and S. L. Pope (1998), "Market Coordination of Transmission Loading Relief Across Multiple Regions," Center for Business and Government, JFK School of Government, Harvard University, Cambridge, Massachusetts.

Jing-Yuan, W. and Y. Smeers (1999), "Spatial Oligopolistic Electricity Models with Cournot Generators and Regulated Transmission Prices," Operations Research 47, 102-112.

Kahn, E. P. (1998), "Numerical Techniques for Analyzing Market Power in Electricity," The Electricity Journal 11, 34-43.

Kinderlehrer, D. and G. Stampacchia (1980), An Introduction to Variational Inequalities and their Applications, Academic Press, New York.

Korpelevich, G. M. (1977), "The Extragradient Method for Finding Saddle Points and Other Problems," Matekon 13, 35-49.

Nash, J. F. (1950), "Equilibrium Points in N-Person Games," Proceedings of the National Academy of Sciences 36, 48-49.

Nash, J. F. (1951), "Noncooperative Games," Annals of Mathematics 54, 286-298. 
Nagurney, A. (1999), Network Economics: A Variational Inequality Approach, Second and Revised Edition, Kluwer Academic Publishers, Dordrecht, The Netherlands.

Nagurney, A. and J. Dong (2002), Supernetworks: Decision-Making for the Information Age, Edward Elgar Publishing, Cheltenham, England.

Nagurney, A., Dong, J. and D. Zhang (2002), "A Supply Chain Network Equilibrium Model," Transportation Research E 38, 281-303.

North American Electric Reliability Council (1998), "Reliability Assessment 1998-2007," September.

Public Utilities Regulating Policies Act, P. L. 95-617 (92 Stat. 3117) 116, U. S. Code Sections 2601.

Schweppe, F. C., Caramanis, M. C., Tabors, R. E. and R. E. Bohn (1988), Spot Pricing of Electricity, Kluwer Academic Publishers, Boston, Massachusetts.

Secretary of Energy Advisory Board's (SEAB) Task Force on Electric System Reliability (1998), "Maintaining Reliability in a Competitive U. S. Electric Industry," September 29.

Singh, H., Editor (1999), IEEE Tutorial on Game Theory Applications in Power Systems, IEEE.

Takriti, S., Krasenbrink, B. and L. S.-Y. Wu (2000), "Incorporating Fuel Constraints and Electricity Spot Prices into the Stochastic Unit Commitment Problem," Operations Research 48, 268-280.

US Energy Information Administration (2002), "The Changing Structure of the Electric Power Industry 2000: An Update."

US-Canada Power System Outage Task Force (2003), "Interim Report: Causes of the August 14th Blackout in the United States and Canada," November.

Wu, F., Varaiya, P., Spiller, P. and S. Oren (1996), "Folk Theorems on Transmission Access: Proofs and Counterexample," Journal of Regulatory Economics 10, 5-24. 
Zaccour, G., Editor (1998), Deregulation of Electric Utilities, Kluwer Academic Publishers, Boston, Massachusetts. 